\title{
T Peak-T End Interval Aggregate
}

National Cancer Institute

\section{Source}

National Cancer Institute. T Peak-T End Interval Aggregate. NCI Thesaurus. Code C117808.

An aggregate T peak-Tend value based on the measurement of T peak-T end from multiple beats within a single ECG. The method of aggregation, which can vary, is typically a measure of central tendency such as the mean. (CDISC) 